\title{
A hierarchical RNN-based model for learning recommendation with session intent detection
}

\author{
Jinyang Liu \\ School of Economics and Management \\ Beihang University \\ Beijing, China \\ liujinyang@buaa.edu.cn
}

\author{
Kunyang Wang \\ Sino-French Engineer School \\ Beihang University \\ Beijing, China \\ wang_ky123@buaa.edu.cn
}

\author{
Chuantao Yin \\ Sino-French Engineer School \\ Beihang University \\ Beijing, China \\ chuantao.yin@buaa.edu.cn
}

\author{
Xiaoyan Zhang \\ Sino-French Engineer School \\ Beihang University \\ Beijing, China \\ xiaoyan.zhang@buaa.edu.cn
}

\author{
Hong Zhou \\ School of Economics and Management \\ Beihang University \\ Beijing, China \\ h_zhou@buaa.edu.cn
}

\begin{abstract}
Since the emergence of MOOCs (Massive Online Open Courses) in the last decade, online education continuously evolves. With the abundance of learning resources provided by MOOC platforms, recommender system can be used to personalize learners' learning experience with respect to learning material consumption. To provide user-adaptive and beneficial recommendation result, the recommender system should be designed with respect to properties of the online learning context, especially the sequential property of learning behaviors. In this paper, we propose a novel model SOLR, a session-based sequential model for online learning material recommendation. We use hierarchical RNN to model online learners' learning sequences on both in-session and cross-session levels. Additionally, attention mechanism is used within sessions to model users' learning session intent. The model is able to learn a hierarchical representation of users' long-term learning history as well as short-term session sequential patterns. We conducted comparative experiments with session-based recommendation baseline methods as well as an ablation study on real-life MOOC dataset. The results show that our model achieves better recommendation results and provide justification for the sequential modeling and model training mechanism implemented in our model.
\end{abstract}

Keywords- online learning, MOOC, recommender system, RNN, smart learning

\section{INTRODUCTION}

Recent years have witnessed the rise of online learning. Since the emergence of MOOCs (Massive Online Open Courses) in the last decade, online education continuously evolves. One of the biggest online learning platforms Coursera had 37 million registered users and over 3100 active courses in 2018 [2]. The abundance of learning resources including digital textbooks, exercises, video tutorials, on-site forums and blogs creates the room and necessity for user personalization and adaptation in online learning systems.
Recommender systems as a form of user personalization play an important role in online services, such as the field of ecommerce, online content consuming (video and music streaming, news, etc. ) and social network. Recommendation techniques are usually classified into three categories: collaborative filtering, content-based and hybrid recommendation models [3]. Collaborative filtering studies the user-item interaction through user behavior or implicit feedbacks and predicts the user's preference towards certain items. Content-based recommender uses item attributes and user profile as auxiliary information to match users with items. Hybrid techniques combine two or more strategies to meet specific requirements of the system [1]. Recently recommender systems using deep neural network gain much attention for their ability to incorporate multiple level of abstraction of the data using neural representation and deep structures and demonstrated great success in their performances [4][5].

There have been successful attempts applying state-of-art recommendation techniques for online learning systems. Collaborative filtering methods have been implemented in early learning management systems [6][7]. Content-based techniques combining with domain ontology such as case-based reasoning and attribute-based matching have been used successfully to recommend learning materials [8]. Although general approaches of recommender systems could be transplanted to online learning platforms, to achieve better performance there are unique challenges to take into consideration within the online learning context [9]:

1. Learning activities are organized in a sequential manner. There's causality in learners' learning histories, i.e. their background knowledge and materials they previously consumed. The sequential and progressive property is intrinsic within education which should be paid more attention to comparing to other recommendation scenarios such as e-commerce and streaming services. 
2. A user's learning path on the platform consists of several sessions. Within each session users demonstrate different learning behavior styles and strategies which should reflect on the materials recommended to them.

To tackle the problems stated above we propose a novel method using session-based sequential recommendation with hierarchical recurrent neural network to capture learners' learning histories as well as their interest within sessions. In addition we use attention mechanism further enhance the session intent. Experiments show that our method achieves state-of-theart performance in online learning dataset.

\section{RELATED WORKS}

The concept of sequential recommendation is primarily used in session-based online activities. For sequential recommendation it's important to capture user's long-term static preference as well as to predict user's short-term behavior in order to recommend the immediate item for user's need [10].

Traditional sequential recommendation techniques include sequential pattern mining, Markov Chains, sequential KNN and session-based matrix factorization. Sequential pattern mining derives from frequent pattern and association rule mining in that it mines a collection of ordered frequent patterns and then performs inference based on predefined support and confidence thresholds [11]. Markov chains (MCs) based methods postulate that the item a user consumes next depends on one or several items he consumed before that. Matrix factorization-based methods are among the most relevant and efficient methods nowadays. Twardowski [12] applied factorization machine to session-based recommendation. Rendle et al. [13] combined first order MC with factorization method and He et al. [14] used similarity matrix factorization combining high order $\mathrm{MC}$ and achieved desirable performance on sparse data.

There are several drawbacks in conventional methods. Sequential pattern mining methods suffer from scalability issues. Besides, they ignore the users' differences in their behavior patterns thus lack the personalization we desire. KNN and matrix factorization have limited ability to capture sequential propriety across sessions. Markov Chain based methods fail to capture users' long-term behavior tendency.

In recent years deep learning with neural networks has achieved great advancement in the field of natural language processing and computer vision. Deep neural models have the ability to incorporate heterogeneous inherent and contextual information of the input with low dimensional representations and reduce the effort of using hand-crafted features. Covington et al. [15] proposed a deep neural model for YouTube video recommendation. Cheng, Heng-Tze, et al. [16] proposed a deepwide neural network structure recommendation framework. Both have achieved significant performance improvement comparing to conventional methods.

Deep neural models that have been adapted to sequential recommendation problems are mainly Recurrent Neural Networks (RNNs) and Convolutional Neural Networks (CNNs). Hidasi et al. proposed GRU4Rec [17], using Gated Recurrent Unit to model the sequential item interactions and training the model using mini-batch parallel training. It's the prototype for several later improved models [18][19]. By utilizing data augmentation strategy and improved pairwise loss function design the GRU4Rec models achieved better performance. The HRNN4Rec model developed by Quadrana et al. [20] first applied hierarchical RNN for recommendation to model users' across-session and inner-session behaviors. Zhang et al. [21] further enriched the item side information by adding dwelling time in RNN recommendation scheme. Compared to RNNs, $\mathrm{CNN}$ structure does better in capturing global and nonconsecutive sequential behavior with lower computational costs. Tang and Wang proposed Caser [22] using convolutional sequence embedding with horizontal and vertical convolutional filters to capture the point-level, union-level and skip-item behaviors.

Attention mechanism was first proposed in natural language processing for machine translation tasks [23]. It models machine's "attention" by assigning different weights to parts of the input sequences which is ideal for modeling short-term intent in sequential recommendation problems. NARM [24] leverages attention mechanism for sessions in its encoder-decoder structure to model user's purpose within sessions. Liu et al. [25] used attention mechanism and calculated the attention correlation between history and recent items.

\section{METHODOLOGY}

In this section, we first frame the session-based recommendation task for online learning. Then we describe our model including the model structure and training specifications.

\section{A. Session-based Recommendation}

A learner's behavior on learning platform consists of several consecutive learning sessions which are loosely defined as the learning material sequences the learner consumes in a period of time. For example, a learner in one session beginning with logging in on the platform could revisit the tutorial video he watched during last session, begin a new video then go to the course forum for further exploration. To describe the problem formally, for a user $\mathrm{u}$ his whole lifetime activities on the platform could be represented as a set of sessions: $S_{u}=$ $\left\{s_{u, 1}, s_{u, 2}, \ldots, s_{u,\left|s_{u}\right|}\right\}$. Each session consists of several learning materials the user consumes: $s_{u, m}=\left\{i_{m, 1}, i_{m, 2}, \ldots, i_{m,\left|s_{m}\right|}\right\}$. A session-based sequential recommender learns the user's sequential behavior and learning history and tries to predict the most suitable material for the user to consume next within the current session.

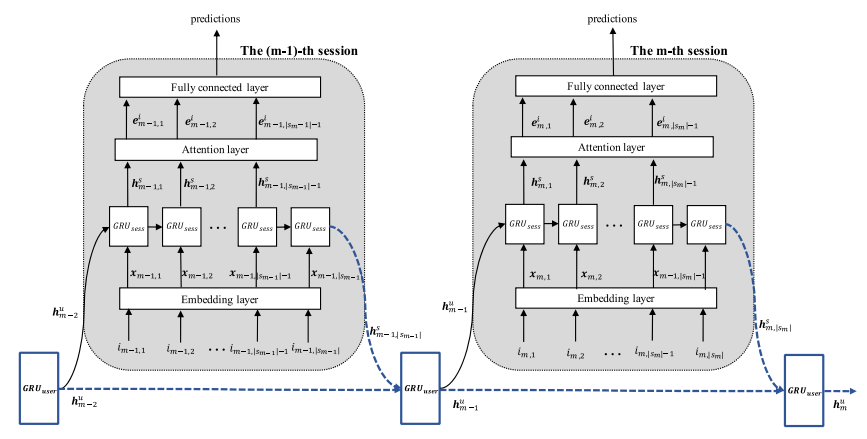

Figure 1. Illustration of the model structure 


\section{B. Model}

To tackle the specific problems of online learning recommendation task, we propose our model Session-based Online Learning Recommender (SOLR). Our model uses a hierarchical recurrent neural network structure with GRU to model users' learning behavior during sessions and their learning history. Attention mechanism is leveraged in the session local encoder to capture users' session intent. The model takes in item embeddings, hierarchically encodes users' local and global sequential behavior. Within sessions the sequence representations are fed into a fully connected layer to produce the recommendation results. The complete model structure is shown in Fig. 1.

Embedding layer We user an attribute-aware embedding layer to produce the item representations taking into account the items' category and type information. The category information could be the learning material's subject and ontology information. The type information is the material's form of its presentation(video, audio, forum thread, etc. ). For an item i, the embedding layer produce the item's representation:

$$
\boldsymbol{x}_{i}=\operatorname{CONCAT}\left(\boldsymbol{e}_{i}^{i}, \boldsymbol{e}_{i}^{c}, \boldsymbol{e}_{i}^{t p}\right)
$$

Where $\boldsymbol{e}_{i}^{i}, \boldsymbol{e}_{i}^{c}$ and $\boldsymbol{e}_{i}^{t p}$ are the embedding of the item ID, category information and type information. The embedding matrices $E^{i} \in \mathbb{R}^{D_{i} \times|I|}, E^{c} \in \mathbb{R}^{D_{c} \times|C|}$ and $E^{t p} \in \mathbb{R}^{D_{t p} \times|T P|}$ each transforms the item's ID, category and type one-hot representation into embedding of dimensions $D_{i}, D_{c}$ and $D_{t p}$.

RNN with GRU A Gated Recurrent Units is a more elaborated model first introduced to tackle the vanishing gradient problems in traditional RNN structure [26]. Using the mechanism of reset and update gates, GRU updates the hidden units in a selective and weighted manners in each step. Compared to GRUs, Long Short Term Memory (LSTM) networks are used more often in NLP tasks. However, [17] shows that replacing GRUs with LSTM led to worse performance in session-based recommendation. The activation of GRU is the interpolation between the previous activation and the candidate activation $\widehat{\boldsymbol{h}}_{t}$ :

$$
\boldsymbol{h}_{\boldsymbol{t}}=\left(1-\boldsymbol{z}_{\boldsymbol{t}}\right) \boldsymbol{h}_{t-1}+\boldsymbol{z}_{\boldsymbol{t}} \widehat{\boldsymbol{h}}_{t}
$$

Where the update gate $\boldsymbol{z}_{\boldsymbol{t}}$ is given by:

$$
\boldsymbol{z}_{t}=\sigma\left(W_{z} \boldsymbol{x}_{t}+U_{z} \boldsymbol{h}_{t-1}\right)
$$

And the candidate activation $\widehat{\boldsymbol{h}}_{t}$ is given by:

$$
\widehat{\boldsymbol{h}}_{\boldsymbol{t}}=\tanh \left(W \boldsymbol{x}_{t}+U\left(\boldsymbol{r}_{t} \odot \boldsymbol{h}_{t-1}\right)\right)
$$

$\boldsymbol{r}_{t}$ is the reset gate and calculated in a similar manner as $\boldsymbol{z}_{\boldsymbol{t}}$ :

$$
\boldsymbol{r}_{t}=\sigma\left(W_{r} \boldsymbol{x}_{t}+U_{r} \boldsymbol{h}_{t-1}\right)
$$

Matrices $W_{z}, U_{z}, W, U, W_{r}$ and $U_{r}$ are all parameters to learn during training. The whole updating process can be noted as: $\boldsymbol{h}_{t}=G R U\left(\boldsymbol{x}_{t}, \boldsymbol{h}_{t-1}\right)$ for simplicity.

Hierarchical RNN The paragraph above describes a classic RNN structure. However, in the context of session-based user behavior, the idea of the sequence of sessions differs from a long item sequence. Users' learning behavior on learning platforms shows different inner-session and cross-session patterns. For example, sessions could be abstracted as learner's taking one or several tutorials, following up with certain points of the lectures, taking exercises or going over all previous sections for refreshment. Each of those groups of activities within sessions differs with respect to the sessions' purposes and behavior patterns. And the progression of sessions signifies the learners' accumulation of knowledge and their achievement on learning paths. Hierarchical RNN was used in HRNN recommender [20] for session-based recommendation. The idea was to build above item sequences (i.e. sessions) RNNs another global RNN encoder to model the session evolution.

The session-level RNN takes within a session each item for input and predicts items the user is consuming next in this session. The user-level RNN takes each session's last hidden state as input, and updates the hidden state for the next session's initialization. We use upper corner $s$ and $u$ to differentiate the hidden state notations in the two levels. As illustrated in Fig. 1, the $\mathrm{m}$-th session initialization is calculated as:

$$
\boldsymbol{h}_{m, 0}^{s}=\tanh \left(W_{\text {init }} \boldsymbol{h}_{m-1}^{u}+\boldsymbol{b}_{\text {init }}\right)
$$

Then within the m-th session, GRU propagates through each step:

$$
\boldsymbol{h}_{m, t}^{s}=G R U_{\text {sess }}\left(\boldsymbol{x}_{t}, \boldsymbol{h}_{m, t-1}^{s}\right)
$$

After the last step's update, the session hidden state is taken as input for the user-level update:

$$
\boldsymbol{h}_{m}^{u}=G R U_{u s e r}\left(\boldsymbol{h}_{m,\left|s_{u, m}\right|}^{s}, \boldsymbol{h}_{m-1}^{u}\right)
$$

Operating on two levels, hierarchical RNN models the innersession dynamics as well as cross-session user evolution which can be justified with the real-life online learning experience.

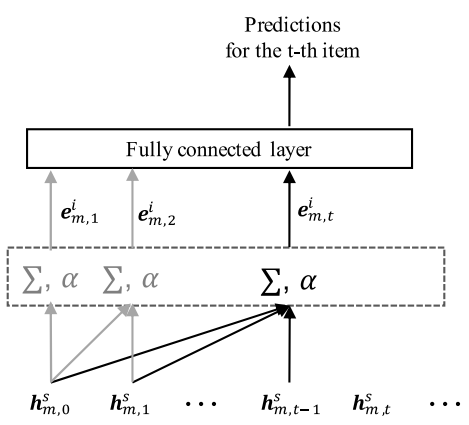

Figure 2. The attention mechanism

Attention layer Attention mechanism is widely used in sequence modeling. During sequence prediction tasks, attention mechanism calculates each input's contribution with respect to the current prediction. In our model during session propagation, to predict the current item we use all previously consumed items to produce a session intent embedding: 


$$
\boldsymbol{e}_{m, t}^{i}=\sum_{j=1, \ldots, t-1} \alpha_{t, j} \boldsymbol{h}_{m, j}^{s}, \quad \text { s.t. } \sum_{j=1, \ldots, t-1} \alpha_{t, j}=1
$$

Where the attention weights $\alpha_{t, j}$ are attained by using the query vector $\boldsymbol{w}^{\alpha}$ with softmax normalization:

$$
\alpha_{\mathrm{t}, \mathrm{j}}=\frac{\exp \left(\mathbf{w}^{\alpha} \mathbf{h}_{\mathrm{m}, \mathrm{j}}^{\mathrm{s}}{ }^{\mathrm{T}}\right)}{\sum_{\mathrm{l}=1, \ldots \mathrm{t}-1} \exp \left(\mathbf{w}^{\alpha} \mathbf{h}_{\mathrm{m}, \mathrm{l}}^{\mathrm{s}}\right)}
$$

As shown in Figure 2, the intent embeddings are fed to the fully connected layer to produce the final prediction for the next item.

\section{Model training}

We use user session parallel mini-batch training with prefix data augmentation method which is well established in the GRU4Rec family [17][18][19]. The user session parallel minibatch training method groups sessions by user then sorts sessions within each group using time stamps. Groups of sessions are fed to the model and trained parallelly. If any of these user sessions end the next sessions are put in their places.

The model is trained with pairwise ranking loss function and negative sampling. The loss function we use is the TOP1 max loss, which is an improvement of the classic TOP1 function by focusing on the most highly ranked negative sample. Given a set of negative samples $N_{S}$, the TOP1 loss function is calculated as:

$$
L_{T O P 1}=\frac{1}{\left|N_{S}\right|} \sum_{j=1, \ldots\left|N_{S}\right|} \sigma\left(r_{j}-r_{i}\right)+\sigma\left(r_{j}^{2}\right)
$$

Where $r_{i}$ and $r_{j}$ are respectively the score of the target item and the score of the negative sample $\mathrm{j}$. The second term in the sum represents a regularization by punishing the high score given to irrelevant items. The TOP1-max loss uses the softmax scores of all negative samples to weight each sample's contribution:

$$
L_{T O P 1-\max }=\frac{1}{\left|N_{s}\right|} \sum_{j=1, \ldots\left|N_{s}\right|} \operatorname{softmax}\left(r_{j}\right) \cdot\left(\sigma\left(r_{j}-r_{i}\right)+\sigma\left(r_{j}^{2}\right)\right)
$$

The loss function focuses on the most wrongly rated items and alleviates the vanishing gradient problem when the number of samples increases [19].

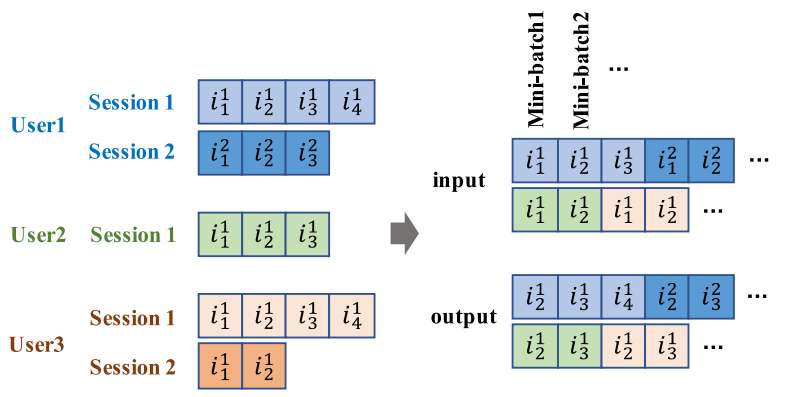

Figure 3. User parallel mini-batch training

We use the user parallel mini-batch training mechanism which is proposed by Quadrana et al. for the HRNN4Rec model. As shown in Figure 3, we group sessions by user then feed several user sequences parallelly to the model. In each iteration, the current mini-batch serves as input for the update of users' $G R U_{\text {sess }}$ and the next mini-batch serves as the ground truth for training. At end of the session, (8) is used for the update of the user's $G R U_{\text {user }}$. If one user's sequence ran out, a new user sequence will be put in its place with $G R U_{\text {user }}$ and $G R U_{\text {sess }}$ reset.

For the negative sampling, the original GRU4Rec model as well as its derived methods including HRNN4Rec all use a mechanism called batch sampling to speed up the training process. Batch sampling takes other parallel sessions' item in the same batch as the negative samples. However, in the case of sequential modeling with explicit user representation, this mechanism suffers from under sampling effect. Because each user sequence can only be exposed to limited parallel users during training. Thus, we opt for the commoner popularity-based sampling mechanism during training.

\section{EXPERIMENTS}

The experiments led in this study consist in two parts: 1 . To demonstrate the effectiveness of our model, we compared our model against five baseline methods for session-based recommendation on their performance in online learning material recommendation. 2 . We realized an ablation study in which we deprived our model of its several functionality modules to show the effectiveness of the mechanisms we implemented in our model.

\section{A. Dataset}

We use the XuetangX dataset of user $\operatorname{logs}$ for our experiments. XuetangX is the largest MOOC platform in China which has provided over 1000 courses and has more than 10 million registered users. The XuetangX dataset contains users' activity logs on the platform from August 2015 to August 2017. There are in total 698 instructor-paced mode (IPM) courses and 515 self-paced mode (SPM) courses. We use users' activity logs for SPM courses since during SPM courses users have more autonomy with respect to learning behaviors. Table I shows the aggregative description for the dataset:

TABLE I. DATASET OVERVIEW

\begin{tabular}{lll}
\hline & Type & Total count \\
\hline $\operatorname{logs}$ & Video activities & $382,225,471$ \\
& Forum activities & 90,815 \\
& Web page activities & $5,496,287$ \\
& Assignment activities & $3,139,558$ \\
& Total materials count & $1,227,078$ \\
\hline \multirow{2}{*}{ enrollment } & Total & 218,274 \\
& Users & 123,719 \\
& SPM courses & 515 \\
\hline
\end{tabular}

\section{B. Baseline methods}

We compare our model against several baseline models:

- Item-KNN: Calculating the item-item cosine similarity based on the co-occurrence of items in sessions across 
users. Regularization is applied to avoid high score. This method is session-based but non-sequential.

- FOSSIL [14]: A hybrid model fusing matrix factorization-based similarity model with high order Markov Chain to take into account both user long-term preference and short-term sequential pattern. In these experiments the maximum order of the Markov Chain is set to 2 .

- GRU4Rec [19]: The improved version of GRU4Rec with prefix and dropout data augmentation mechanism and loss function with top-k gains. This model doesn't have user representations thus lacks personalization.

- HRNN4Rec [20]: The hierarchical RNN model for session recommendation. It could be seen as our model without attention mechanism. Similar to the original model, the model is trained with user parallel mini-batch training. But we replace the original batch sampling mechanism with popularity-based negative sampling.

- u-GRU4Rec: A modified GRU4Rec model with recurrent user representation by joining sessions of the same user into a long sequence. Same to HRNN4Rec the model is trained with user parallel mini-batch training with popularity-based negative sampling.

\section{Experimental setups}

For our model, the item embedding, session hidden state and user hidden state are respectively of size 100, 256 and 256. For item embedding we include a category embedding using the course label to which the items belong. We train the model with user session parallel mini-batch training with batch size 500 . The uniform negative sampling size is set to 1024 .

For other neural network baseline methods, we use the same setup for the size of hidden state vectors and training parameters. For the two models with user representation (u-GRU4Rec and HRNN4Rec) we didn't implement the batch sampling method used in their original methods for its negative effect of under sampling. Instead we used uniform sampling as used in our model with the same sampling size.

We use all users' last session as test data and all their previous sessions as training data. The evaluation is also carried in a user parallel fashion.

\section{Results and analysis}

General performance As shown in Table II, our model outperforms the baseline models in recall and ranking evaluations. The following observations can be made:

Compared to the baseline methods' performances on other recommendation datasets such as movie and e-commerce datasets in their original studies, the recall and ranking evaluations are rather high across models for learning material recommendation for XuetangX dataset. This can be interpreted as the unique behavior paradigm on online learning platforms. Users' behavior sessions are more or less predefined by the way the materials are organized. When an online course is curated by a MOOC provider it's usually segmented by several learning sessions i.e. lessons. Users usually follow the material sequences within the paths of the courses in most part of the learning activity. Most of the uncertainty happens when users drift away from the course pages and into the forum threads as well as the revisiting of former consumed materials across several sessions.

TABLE II. General PERFormance RESUlt

\begin{tabular}{lcccc}
\hline \multirow{2}{*}{ Methods } & \multicolumn{2}{c}{ Recall } & \multicolumn{2}{c}{ Ranking } \\
& HR@5 & HR@10 & MRR@5 & MRR@10 \\
\hline Item-KNN & 0.2581 & 0.3012 & 0.1403 & 0.2068 \\
FOSSIL & 0.3492 & 0.4607 & 0.2711 & 0.2901 \\
GRU4Rec & 0.3601 & 0.4613 & 0.2840 & 0.3006 \\
u-GRU4Rec & 0.4364 & 0.5370 & 0.2995 & 0.3470 \\
HRNN4Rec & 0.4388 & 0.5482 & 0.3010 & 0.3501 \\
SOLR & $\mathbf{0 . 4 5 6 5}$ & $\mathbf{0 . 5 5 2 1}$ & $\mathbf{0 . 3 1 7 2}$ & $\mathbf{0 . 3 7 5 4}$ \\
\hline
\end{tabular}

Compared to non-sequential baseline model item-KNN, the sequential recommendation models have significant higher scores. This proves that online learning activities are inherently sequential thus justifies the methodology of using sequential recommendation techniques in the online learning domain.

Compared to non-neural methods(Item-KNN and FOSSIL), the three neural network methods have better performances on both ranking and recall evaluation metrics which proves that recurrent neural networks are better apt to sequential modeling.

Models with recurrent user representation(u-GRU4Rec, HRNN4Rec and our model) outperform GRU4Rec model which is a non-personalized session-based sequential method. This could be attributed to the differences of the sequential patterns with respect to personal learning style and preference in the learning behaviors among users. Models with user representation are able to incorporate these personalized sequential patterns into user-distinct recurrent states. Also HRNN4Rec and our model both have better performances than u-GRU4Rec which shows the benefit of the hierarchical recurrent user representation. The traditional RNN structure is proved to have difficulty in long sequence modeling. The HRNN structure uses cross-session level update to aggregate the sequential history and in this case is more fitting to the online learning scenario.

Overall our model outperformed all the baseline methods on both recall and ranking evaluation metrics. This could be attributed to the contribution of the user-level global representation and the contribution of attention mechanism within session.

In-session performance analysis We compared the four sequential recommendation models above with user representation(FOSSIL, u-GRU4Rec, HRNN4Rec and our model) in their session-level performances by breaking down the sequential session recommendation into three stages: beginsession, mid-session and the end-session. For begin-session performance we evaluate the recall and ranking ability of each model on the first two item of the session. The end-session is seen as the last item in the session and the rest is the mid-session. 
From the results shown in Table III, We can see that our model and HRNN4Rec have better performance in beginsession recommendation due to the session initialization using hierarchical user recurrent representation. With user representation the model can use the user's learning history information and cross-session behavior to predict the beginning of the user's next session. In mid-session and end-session recommendation our model has the best performance among the four, which is a testament to the session intent detection ability of the attention mechanism.

TABLE III. SESSION LEVEL PERFORMANCE RESULTS

\begin{tabular}{|l|ccc|ccc|}
\hline \multirow{2}{*}{ Models } & \multicolumn{3}{|c|}{ HR@5 } & \multicolumn{3}{c|}{ MRR@5 } \\
& begin & mid & end & begin & mid & end \\
\hline FOSSIL & 0.2041 & 0.3510 & 0.3587 & 0.1408 & 0.2782 & 0.2815 \\
u-GRU4Rec & 0.2603 & 0.4395 & 0.4497 & 0.1579 & 0.2996 & 0.3105 \\
HRNN4Rec & 0.3550 & 0.4406 & 0.4404 & 0.2071 & 0.3006 & 0.3230 \\
SOLR & $\mathbf{0 . 3 5 5 6}$ & $\mathbf{0 . 4 5 3 1}$ & $\mathbf{0 . 4 5 8 7}$ & $\mathbf{0 . 2 0 7 5}$ & $\mathbf{0 . 3 1 4 1}$ & $\mathbf{0 . 3 3 5 4}$ \\
\hline
\end{tabular}
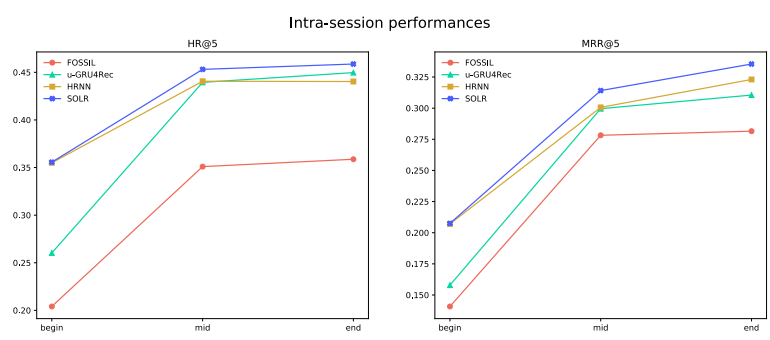

Figure 4. Session level performance comparison

The effect of the length of user learning history To further illustrate our model's strength in users' learning sequence modeling we inspected the performances of the neural network models with respect to the length of users' learning history. The users were regrouped into three categories: users with short, medium and long learning history which contains respectively users with under 10, 10 to 30 and over 30 learning sessions. The models put in comparison are u-GRU4Rec, HRNN4Rec and our model, with the non-personalized GRU4Rec for the control group.

The results show that $\mathrm{u}-\mathrm{GRU} 4 \mathrm{Rec}$ with the traditional RNN structure has declined performance for users with longer learning history. In this case considering the average items in one sessions of the XuetangX user logs, the normal RNN structure has difficulty in modeling learning material sequences after 200 updates on average. However both HRNN4Rec and our model show the increase in performance with the growth in length of user history, with our model achieving higher performance especially in ranking metric.

We reason that the hierarchical RNN structure's update mechanism is close to nature of user's progression of their learning activities. Using HRNN the models have a better ability in modeling the accumulation and abstraction of learning history.
TABLE IV. EFFECTS OF LENGTH OF HISTORY

\begin{tabular}{|l|ccc|ccc|}
\hline \multicolumn{1}{|c|}{ Models } & \multicolumn{3}{|c|}{ HR@5 } & \multicolumn{3}{c|}{ MRR@5 } \\
& short & medium & long & short & medium & long \\
\hline GRU4Rec & 0.3618 & 0.3582 & 0.3601 & 0.2837 & 0.2841 & 0.2830 \\
u-GRU4Rec & 0.4025 & 0.4382 & 0.4309 & 0.2989 & 0.3012 & 0.2932 \\
HRNN4Rec & 0.4101 & 0.4390 & 0.4516 & 0.2972 & 0.3005 & 0.3054 \\
SOLR & $\mathbf{0 . 4 2 3 5}$ & $\mathbf{0 . 4 5 2 5}$ & $\mathbf{0 . 4 6 2 8}$ & $\mathbf{0 . 2 9 9 6}$ & $\mathbf{0 . 3 1 2 2}$ & $\mathbf{0 . 3 2 4 1}$ \\
\hline
\end{tabular}
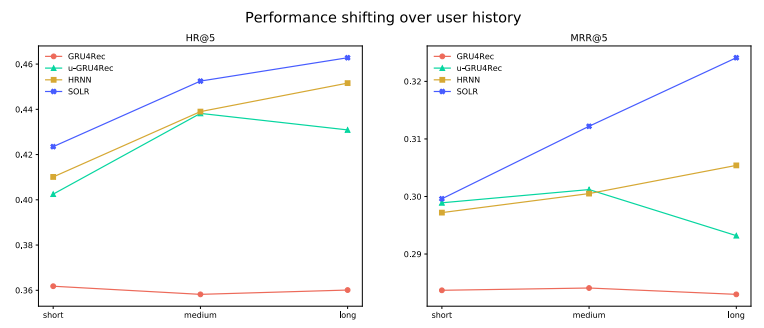

Figure 5. Performance comparison with different user history length

\section{E. Ablation study}

To demonstrate the effectiveness of our design of the model functionalities and choices for training mechanisms, we realized an ablation study where three mechanisms in the model are inspected for their contribution to recommendation performance: the attention mechanism, item embedding with extrainformation and the popularity sampling mechanism. In each experiment we remove one of the mechanism and keep the other two then train and evaluate the model with the dataset.

\begin{tabular}{|c|c|c|c|c|c|}
\hline \multicolumn{4}{|c|}{ Methods } & \multirow{2}{*}{$\begin{array}{l}\text { Recall } \\
\text { HR@5 }\end{array}$} & \multirow{2}{*}{$\begin{array}{r}\text { Ranking } \\
\text { MRR@5 }\end{array}$} \\
\hline & attention & $\begin{array}{c}\text { extra } \\
\text { embed. }\end{array}$ & sampling & & \\
\hline $\begin{array}{l}\text { SOLR } \\
\text { w/o attention }\end{array}$ & - & + & popularity & $\begin{array}{c}0.4421 \\
(-3.15 \%)\end{array}$ & $\begin{array}{c}0.3056 \\
(-3.66 \%)\end{array}$ \\
\hline $\begin{array}{l}\text { SOLR } \\
\text { w/o } \\
\text { popularity sampling }\end{array}$ & + & + & batch & $\begin{array}{c}0.4404 \\
(-3.61 \%)\end{array}$ & $\begin{array}{c}0.2811 \\
(-11.4 \%)\end{array}$ \\
\hline $\begin{array}{l}\text { SOLR } \\
\text { w/o } \\
\text { extra embedding }\end{array}$ & + & - & popularity & $\begin{array}{c}0.4522 \\
(-1.02 \%)\end{array}$ & $\begin{array}{c}0.3106 \\
(-2.08 \%)\end{array}$ \\
\hline SOLR & + & + & popularity & 0.4565 & 0.3172 \\
\hline
\end{tabular}

As shown in Table V, when the popularity-based sampling is changed to batch sampling used by the original GRU4Rec series of models, the model suffers from the most severe decline. This is due to the under sampling effect of using batch sampling method in user parallel mini-batch training. The model without the attention mechanism also has performance decline which is expected according to the comparison between our model and HRNN4Rec. However, the extra-information embedding with 
course information and material type information doesn't appear to have a big effect in recommendation performance. We reason that this is due to the particular behavior patterns of online learning with respect to course subject and material type. Usually the learning materials are organized by curators and educators such that users can follow through the predetermined learning path. And most users feel comfortable to do as such. Most of the randomness appearing in learning sequences is in session beginning and when users go to materials out of course structure such as wiki pages and forum threads. Thus most of the sequential information with respect to the learning materials' course and type information is learnt during training with only the embedding of item IDs. Overall the experimentation results justified the mechanisms used in our model. The HRNN structure combined with in-session attention mechanism, extrainformation embedding and popularity-based sampling mechanism has better ability in sequential recommendation and more adaptive to the online learning context.

\section{CONCLUSION}

This paper presented a hierarchical recurrent neural network model with attention mechanism for learning material recommendation on online learning platform. It captures users' learning history and session behavior patterns in both global and session-level. Experiments conducted on real life data from XuetangX MOOC platform demonstrate the effectiveness of our model by comparing it to other sequential session-based recommendation methods. The comparisons of the performances on session-level and with various user history lengths show that our model is capable to incorporate long-term user behaviors and to learn an aggregated representation of user history. By modeling users' intent during a session using attention mechanism, the model can achieve better performance for recommendation within current session. The experiment results prove that our model has better compatibility with the highly sequential online learning behavioral context. We would further explore the potential of incorporating more material side information such as tag information and domain ontology which we believe can contribute to session-based recommendation for online learning. The model could also be applied in other domains of recommendation where the user behavior has an inherently sequential property.

\section{ACKNOWLEDGEMENT}

This work is supported by the National Natural Science Foundation of China (No. 61977003).

\section{REFERENCES}

[1] Gediminas Adomavicius and Alexander Tuzhilin. 2005. Toward the next generation of recommender systems: A survey of the state-of-the-art and possible extensions. IEEE transactions on knowledge and data engineering 17, 6 (2005), 734-749.

[2] By The Numbers: MOOCs in 2018. https://www.classcentral.com/report/mooc-stats-2018/

[3] Dietmar Jannach, Markus Zanker, Alexander Felfernig, and Gerhard Friedrich. 2010. Recommender systems: an introduction.
[4] Li Deng, Dong Yu, and others. 2014. Deep learning: methods and applications. Foundations and Trends $®$ in Signal Processing 7, 3-4 (2014) 197-387.

[5] Zhang, Shuai, et al. "Deep learning based recommender system: A survey and new perspectives." ACM Computing Surveys (CSUR) 52.1 (2019): 5.

[6] Recker, M. M., Walker, A., \& Lawless, K. (2003). What do you recommend? Implementation and analyses of collaborative information filtering of web resources for education. Instructional Science, 31(4-5), 299-316.

[7] Lynch, C., Ashley, K., Aleven, V., \& Pinkwart, N. (2006). Defining illdefined domains; a literature survey. In Proceedings of the workshop on intelligent tutoring systems for ill-defined domains at the 8th international conference on intelligent tutoring systems (pp. 1-10)

[8] Heraud, J.-M., France, L., \& Mille, A. (2004). Pixed: An ITS that guides students with the help of learners' interaction log. In International conference on intelligent tutoring systems, workshop analyzing student tutor interaction logs to improve educational outcomes. Maceio, Brazil (pp. 57-64).

[9] Klašnja-Milićević, Aleksandra, et al. E-learning systems: Intelligent techniques for personalization. Vol. 112. Springer, 2016.

[10] S. Wang, L. Cao, and Y. Wang, "A survey on session-based recommender systems," arXiv preprint arXiv:1902.04864, 2019.

[11] N. R. Mabroukeh and C. I. Ezeife, "A taxonomy of sequential pattern mining algorithms," ACM Comput. Surv., vol. 43, no. 1, pp. 1-41, 2010.

[12] B. Twardowski, "Modelling contextual information in session-aware recommender systems with neural networks," in RecSys, 2016, pp. 273276.

[13] S. Rendle, C. Freudenthaler, and L. Schmidt-Thieme, "Factorizing personalized markov chains for next-basket recommendation," in WWW, 2010, pp. 811-820.

[14] R. He and J. McAuley, "Fusing similarity models with markov chains for sparse sequential recommendation," in ICDM, 2016, pp. 191-200.

[15] Covington, Paul, Jay Adams, and Emre Sargin. "Deep neural networks for youtube recommendations." Proceedings of the 10th ACM conference on recommender systems. ACM, 2016.

[16] Cheng, Heng-Tze, et al. "Wide \& deep learning for recommender systems." Proceedings of the 1st workshop on deep learning for recommender systems. ACM, 2016.

[17] B. Hidasi, A. Karatzoglou, L. Baltrunas, and D. Tikk, "Session-based recommendations with recurrent neural networks," arXiv preprint arXiv:1511.06939, 2015.

[18] Y. K. Tan, X. Xu, and Y. Liu, "Improved recurrent neural networks for session-based recommendations," in DLRS, 2016, pp. 17-22.

[19] B. Hidasi and A. Karatzoglou, "Recurrent neural networks with top-k gains for session-based recommendations," in CIKM, 2018, pp. 843-852.

[20] M. Quadrana, A. Karatzoglou, B. Hidasi, and P. Cremonesi, "Personalizing session-based recommendations with hierarchical recurrent neural networks," in RecSys, 2017, pp. 130-137.

[21] Y. Zhang, H. Dai, C. Xu, J. Feng, T. Wang, J. Bian, B. Wang, and T. Liu, "Sequential click prediction for sponsored search with recurrent neural networks," national conference on artificial intelligence, pp. 1369-1375, 2014.

[22] J. Tang and K. Wang, "Personalized top-n sequential recommendation via convolutional sequence embedding," in WSDM, 2018, pp. 565-573.

[23] D. Bahdanau, K. Cho, and Y. Bengio, "Neural machine translation by jointly learning to align and translate," ICLR, 2015.

[24] J. Li, P. Ren, Z. Chen, Z. Ren, T. Lian, and J. Ma, "Neural attentive session-based recommendation," in CIKM, 2017, pp. 1419-1428.

[25] Q. Liu, Y. Zeng, R. Mokhosi, and H. Zhang, "Stamp: short-term attention/memory priority model for session-based recommendation," in SIGKDD, 2018, pp. 1831-1839.

[26] Cho, K., Van Merriënboer, B., Bahdanau, D. and Bengio, Y., 2014. On the properties of neural machine translation: Encoder-decoder approaches. arXiv preprint arXiv:1409.1259. 